\title{
EXPERIMENTS ON VORTEX-INDUCED VIBRATION OF A VERTICAL CYLINDRICAL STRUCTURE: EFFECT OF LOW ASPECT RATIO
}

\author{
M.A.A. Rahman ${ }^{1,2 *}$, and K.P. Thiagarajan ${ }^{3}$ \\ ${ }^{1}$ School of Ocean Engineering, Universiti Malaysia Terengganu, \\ 21030 Kuala Terengganu, Terengganu, Malaysia \\ ${ }^{2}$ School of Mechanical and Chemical Engineering, \\ University of Western Australia, Perth, Australia 6009. \\ Email: mohdasamudin@umt.edu.my \\ Phone: +6096683698; Fax: +096683991 \\ ${ }^{3}$ Department of Mechanical Engineering, University of Maine, \\ Orono, USA 04469.
}

\begin{abstract}
Studies in offshore engineering have shown that vortex-induced vibration (VIV) of a slender offshore riser can be fundamentally different from that of a cylindrical spar structure. Under similar environments, the primary difference between the two structures affecting vortex shedding relates to the aspect ratio (length to diameter ratio, $L / D)$. In the present investigation, careful experiments were carried out on the VIV of an elastically mounted cylinder with a varying aspect ratio. Experiments were conducted by forcing the vertical cylinders in still water. Of particular interest was the measurement of the response amplitude, hydrodynamic forces, Strouhal number (St) and the lock-in region while the aspect ratio of the cylinder varied from $L / D=0.5$ to $L / D=13$. The experiments were conducted in the subcritical flow region $\left(7.4 \times 10^{3}<\right.$ $R e<2 \times 10^{5}$ ), corresponding to a range of reduced velocity $U_{r}$ from 2 to 14 . Comparisons with experimental results obtained from the literature showed meaningful trends. It was found that the response amplitude of the structure decreased as the aspect ratio was reduced. The decrease in response amplitude was accompanied by a decrease in the correlation length of the vortex shedding and the hydrodynamic forces experienced by the structure. The Strouhal number and lock-in region, too, showed a significant reduction with a lower aspect ratio. It is apparent that the aspect ratio influenced the occurrence of the VIV, which affected the hydrodynamics of the fluidstructure interaction between the fluid and the bluff body.
\end{abstract}

Keywords: Vortex-induced vibration; free vibration experiment; aspect ratio effect; cylindrical offshore structures; circular FPSO; classic spar.

\section{INTRODUCTION}

Many offshore structures situated in the sea are subjected to severe current conditions. These include fixed offshore structures, risers, mooring lines, floating structures, and pipelines, which undergo vortex-induced vibration (VIV) during daily operations. The interaction of the fluid and the structure can produce a potentially destructive force on the structure itself. VIV is a phenomenon caused by the shedding of the vortices from bluff bodies in a fluid flow. The shedding generates forces which compel bodies to undergo periodic oscillation and normally causes large stresses and fatigue on the 
structure. This topic has been extensively reviewed by King [1], Sarpkaya [2, 3], Griffin [4], Bearman [5], Pantazopolous [6], Williamson and Govardhan [7, 8], and is also the subject of books by Chen [9], Blevins [10], and Sumer and Fredsoe [11], to name but a few.

Recently, various types, sizes, shapes, and lengths of offshore structures have been installed which are predisposed to problems arising from vortex shedding. One of the important factors influencing VIV is the change of the structure aspect ratio $(L / D)$. The study is focused on the particular scenario of circular floating structures with varying $L / D$ such as spar platforms, floating production storage and offloading (FPSO) and many other circular cylindrical structures. In practice, the typical height of the spar platform in the Gulf of Mexico (GOM) varies from 142 to $230 \mathrm{~m}$ with a hull diameter of 27 to $45 \mathrm{~m}$, corresponding to $L / D$ between 4 and 10 . A classic spar consists of a long cylindrical hull with almost $90 \%$ of the hull submerged in the water [12]. In a circular FPSO, as illustrated in Figure 1, the overall length of the platform is $66 \mathrm{~m}$ with a hull diameter of approximately $60 \mathrm{~m}$. This circular FPSO is the world's first cylinder-shaped FPSO and yields $L / D \approx 1.1$. Its unique design and technology provide improved motion, higher stability reserves, and a higher deck load capacity than a conventional FPSO [13].

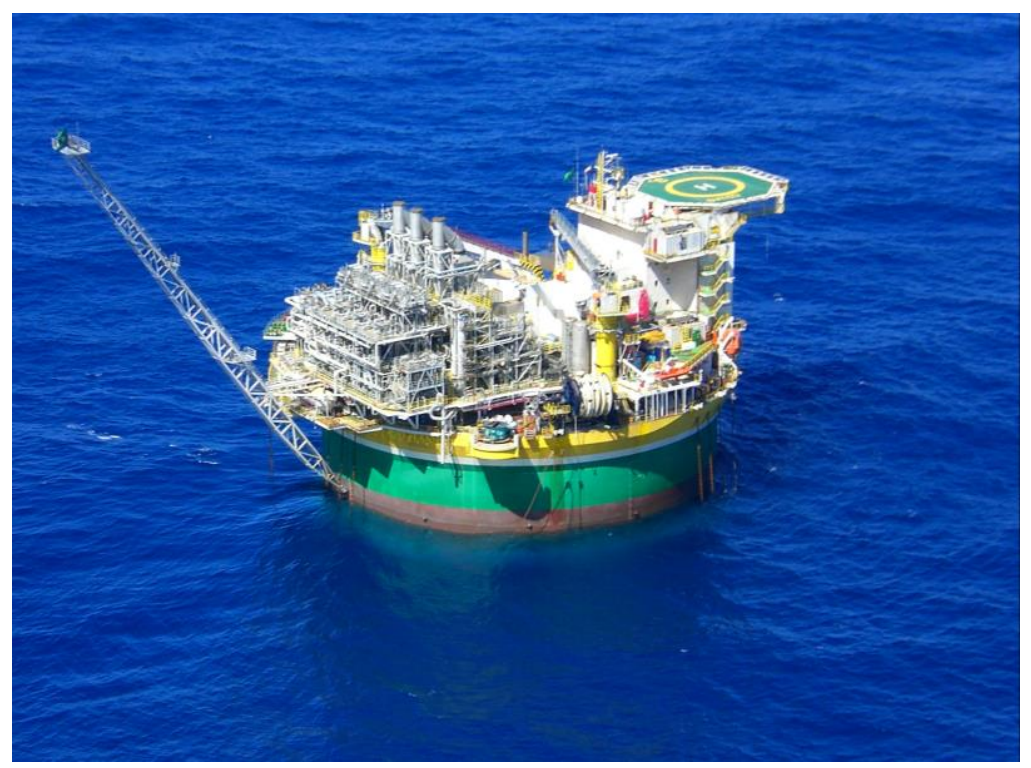

Figure 1. Sevan Piranema, the first Sevan circular-shaped FPSO.

The influence of $L / D$ on VIV response around a circular cylinder was investigated by Nishioka and Sato [14], West and Apelt [15], Lee and Budwig [16], Baban and So [17], Szepessy and Bearman [18], Albarede and Monkewitz [19], Norberg [20], and Nakamura et al. [21]. Most of these researchers conducted experiments in wind tunnel facilities. To the authors' knowledge, there are only a few studies on the effects of $L / D$ which have been conducted in water channels for low cylinder aspect ratio (e.g. $L / D<10$ ). For some of the latest work in this line, see Goncalves et al.[22]. Changes in aspect ratio are expected to alter the morphology of the wake region and at the same time affect pressure and amplitude. A low pressure was normally measured in the wake region during VIV tests. The pressure significantly changed when the aspect ratio changed. Clearly, it can be suggested that the amplitude of the vibrations is proportional to the correlation length of the VIV, based on the 
variation of pressure distribution on the structure. King [1] investigated the value of the correlation length over a range of Reynolds numbers and showed that the correlation length decreases with increasing Reynolds number. He also observed that the end conditions had an effect on low aspect ratio cases compared with the infinite length cylinder. In the present experiments, the effects of the free-end conditions and the free surface were captured purposely to simulate real floating offshore platform situations (i.e. spars and circular-type FPSO). Recent experiments on the free surface of vertical cylinders were performed by Rahman et al. [23] which show the disturb closer effects as the cylinder comes close to the free surface. In the present work, the effects of the aspect ratio $(L / D)$ on the VIV response for a free oscillating cylinder in a uniform flow were studied. The aspect ratio investigated in this study varies from $L / D=0.5$ to $L / D=$ 13 and covers most of the range intended for particular classic spar and circular-type FPSOs. Endplates were deliberately not used because of our interest in simulating real floating offshore platforms such as spar platforms and circular-type FPSOs. The collected data for the normalized amplitude thus include the effects of the end conditions (3D effects) for each aspect ratio examined. We believe that the aspect ratio effects on structural behavior become significant in the case of VIV in real sea conditions. Thus, the study of the effect of a structure's aspect ratio on hydrodynamic forces and responses is crucial and can contribute to a better understanding of the issue. The significance of this study is its contribution of new results for experimental investigation of a range of low aspect ratios in water without the use of end plates.

\section{EXPERIMENTAL SETUP}

\section{Design of Experiment}

Free vibration experiments were carried out in the Hydraulic Tank Facility, School of Environmental System Engineering (SESE) at the University of Western Australia. The free vibration experimental apparatus consisted of four sections; the towing tank, experimental rig, tow carriage, and the towing mechanism as illustrated in Figure 2. The towing tank was $50 \mathrm{~m}$ long, $1.25 \mathrm{~m}$ wide and $1.1 \mathrm{~m}$ deep. The experimental rig (Figure 3) consisted of a pendulum system and a frame rig. The pendulum system was modeled by four arms, and was attached to the base plate and the cylinder. The cylinder was mounted vertically on the four-armed pendulum system. Universal joints were used on each pendulum arm's connection to ensure a smooth swing of the pendulum. This setup was attached to the experimental frame rig and then positioned on top of the tow carriage in the fluid channel. The tow carriage was equipped by a motor and data acquisition system. The towing mechanism used on the tank was powered by an electric motor (ASEA model, 3.9kW, and 1520rpm). A continuous cable drive for the towing system was used as it can be controlled to run at a range of velocities. An analogue speed controller was used to control the velocity of the carriage over a range $0<U<3.5$ $\mathrm{m} / \mathrm{s}$. The towing speed was calibrated with a tachometer in order to minimize errors during experiments.

The experiments were focused on a single degree of freedom only, i.e. along the transverse direction of the fluid flow. Hence, the motion of the cylinder in the inline direction was neglected in this study. The pendulum arms were restrained in the flow direction (inline) by installation of braces on the pendulum arms in the parallel direction. The experiments were conducted in the subcritical flow region $\left(7.4 \times 10^{3}<R e\right.$ $<2 \times 10^{5}$ ), corresponding to a range of reduced velocity $U_{r}$, from 2 to 14 . In order to 
simulate a uniform steady current condition, the experimental rig was attached to the towed carriage and towed through still water.

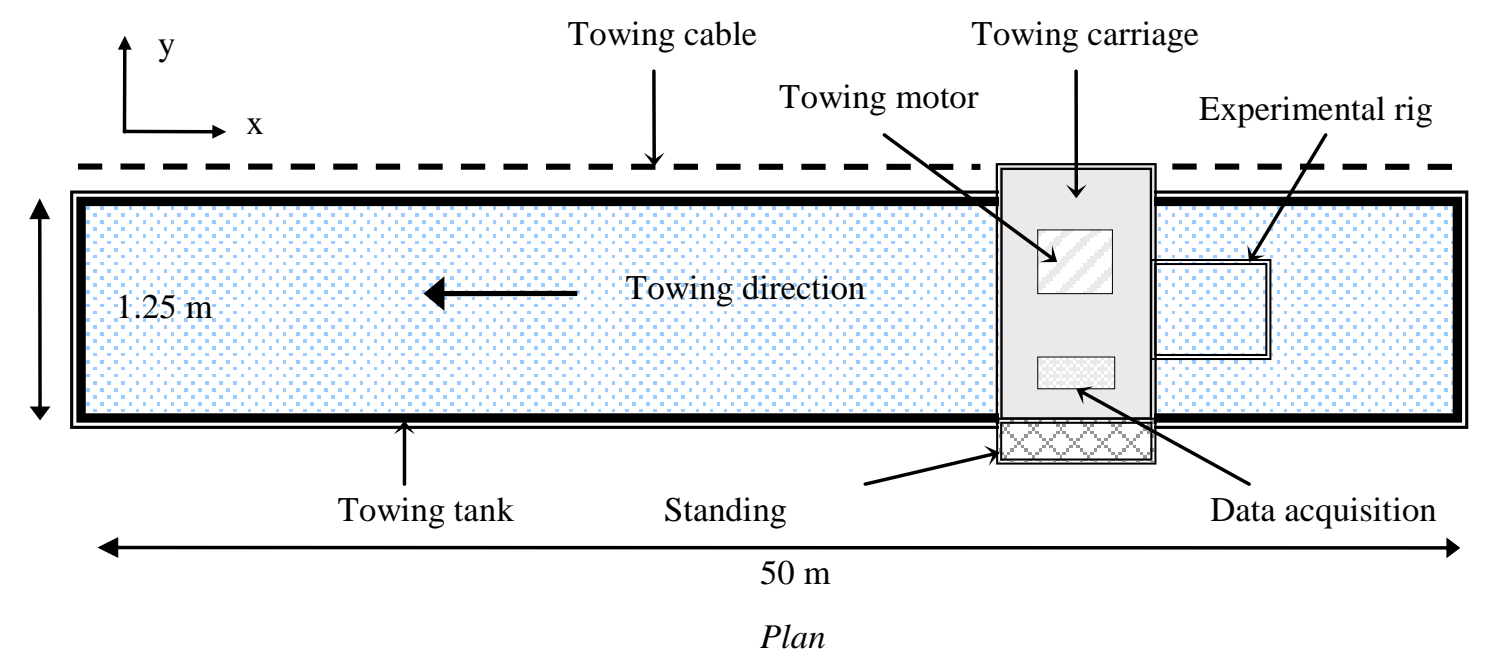

Figure 2. Hydraulic tank at University of Western Australia.

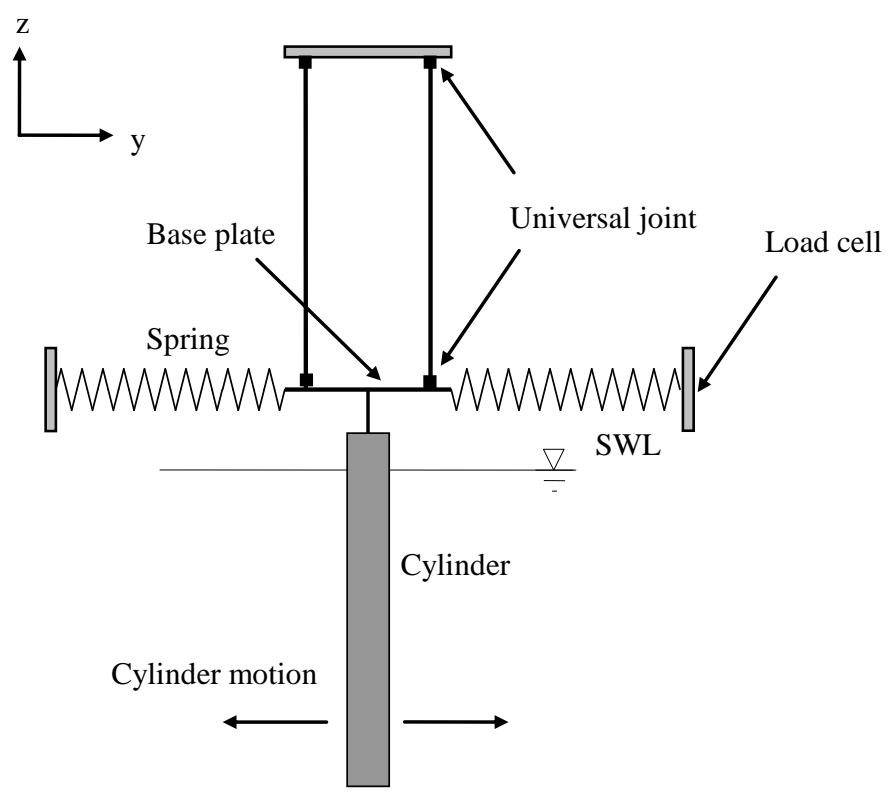

Figure 3. Schematic diagram of the pendulum rig used for the experiments.

Two load cells were fitted in the transverse direction on the experimental rig. Two linear springs connected the cylinder in the transverse direction to load cells. The springs were preloaded in order to obtain linear translations over an entire cycle of oscillation. The cylinder transverse displacement was evaluated from the load cell measurement by using calibrated values of spring stiffness. The maximum amplitude defined by Stappenbelt et al. [24] as the mean of the largest 10\% half-peak to trough values was used throughout this test. Besides cylinder displacement, the quantities measured during the experiments were drag and lift forces. These hydrodynamic forces were measured by a set of strain gages in inline and transverse directions. The strain gages were fitted on a rod connected to the cylinder (Figure 4). The signals from the 
load cells and strain gages were sampled simultaneously from 60 to 120 seconds at a sampling rate of $100 \mathrm{~Hz}$. The test duration depended on the distance towed along the tank corresponding to the carriage velocity. The time-dependent data were processed in a MATLAB ${ }^{\mathrm{TM}}$ environment to obtain the amplitudes of motion and forces, as well as their dominant frequencies.

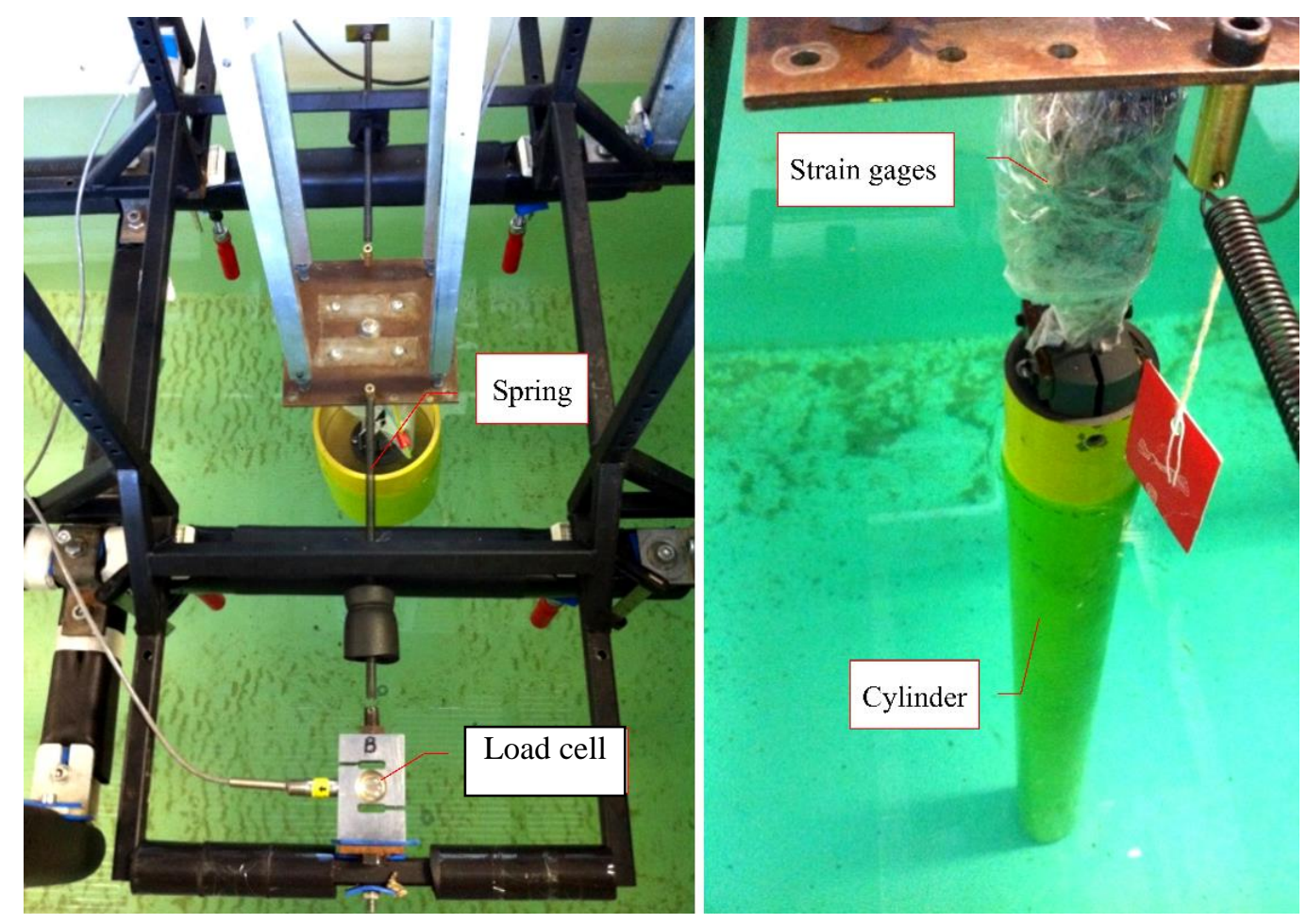

Figure 4. Experimental setup for the investigation of VIV on different aspect ratios.

\section{Experimental Procedure}

The experiments consisted of eight different aspect ratios with four different cylinder diameters. The tests were conducted by one-way towing direction in all cases. The bottom end was fitted with a cap to ensure watertightness. The cylinder models were made from PVC, designed as a smooth cylinder, so no roughness effects were considered. The draft of the cylinder varied from $0.05 \mathrm{~m}$ to $0.25 \mathrm{~m}$ thus exposed to a free surface effect and wave run up on the structure. The structural damping and natural frequency of the cylinders were evaluated by free decay tests conducted prior to each aspect ratio experiment as practiced by Stappenbelt et al. [24]. The free decay test for each corresponding aspect ratio was repeated five times and the average value was calculated. Table 1 shows the value of the natural frequencies and the damping ratios in still water. The damping values were observed to vary between $2.1 \%$ and $3.6 \%$ and the natural frequencies from 0.508 to $1.120 \mathrm{~Hz}$. The natural frequencies for high aspect ratio cylinders ( $L / D=5-13$ ) were slightly higher (around $38 \%$ to $55 \%$ ) than those of the low aspect ratio cylinders $(L / D=0.5$ to 3$)$. 
Table 1. Experimental parameters.

\begin{tabular}{cccccccc}
\hline $\begin{array}{c}\text { Exp. } \\
\text { no. }\end{array}$ & $\begin{array}{c}L \\
(\mathrm{~m})\end{array}$ & $\begin{array}{c}D \\
(\mathrm{~m})\end{array}$ & $L / D$ & $\begin{array}{c}m \\
(\mathrm{~kg})\end{array}$ & $\begin{array}{c}m_{b} \\
(\mathrm{~kg})\end{array}$ & $\begin{array}{c}m_{f} \\
(\mathrm{~kg})\end{array}$ & $\begin{array}{c}k \\
(\mathrm{~N} / \mathrm{m})\end{array}$ \\
\hline 1 & 0.78 & 0.06 & 13 & 1.886 & 1.642 & 2.205 & 245 \\
2 & 0.6 & 0.06 & 10 & 1.886 & 0.828 & 1.696 & 245 \\
3 & 0.6 & 0.08 & 7.5 & 1.925 & 2.900 & 3.016 & 245 \\
4 & 0.4 & 0.08 & 5 & 1.925 & 1.292 & 2.011 & 245 \\
5 & 0.33 & 0.11 & 3 & 2.351 & 2.667 & 3.136 & 52 \\
6 & 0.22 & 0.11 & 2 & 2.351 & 0.994 & 2.091 & 52 \\
7 & 0.16 & 0.16 & 1 & 2.438 & 2.709 & 3.217 & 52 \\
8 & 0.08 & 0.16 & 0.5 & 2.438 & 0.135 & 1.608 & 52 \\
\hline
\end{tabular}

The mass ratio defined in this present work is the ratio of the total oscillating mass, $m_{o s c}$, to the displaced fluid mass, $m_{f}$

$$
m^{*}=m_{o s c} m_{f}^{-1}
$$

where the oscillating mass is the total mass of the structure, $m_{s}$ includes enclosed fluid, $m_{e n c}$, but excludes the hydrodynamic mass, $m_{a}$, as defined by Stappenbelt et al. [24]. The mass ratio of the experiments was kept constant at a value of 1.6. This was achieved by adding the ballast mass $m_{b}$ (sand) to the total structural mass, $m$, for each test case, using the formulation of

$$
m_{o s c}=m+m_{b}
$$

and the displaced fluid mass was obtained by

$$
m_{f}=0.25 \rho \pi D^{2} L
$$

where $\rho$ is the density of the fluid, and $D$ and $L$ are the diameter and the length of the cylinder respectively. The experimental parameters used in the present study are listed in Table 2.

Table 2. Natural frequencies and damping value in still water.

\begin{tabular}{clll}
\hline Exp. no. & $L / D$ & $\begin{array}{c}f_{n \text { still water }} \\
(\mathrm{Hz})\end{array}$ & $\zeta_{\text {still water }}$ \\
\hline 1 & 13 & 0.990 & 0.031 \\
2 & 10 & 1.120 & 0.031 \\
3 & 7.5 & 0.865 & 0.036 \\
4 & 5 & 1.035 & 0.032 \\
5 & 3 & 0.508 & 0.026 \\
6 & 2 & 0.569 & 0.021 \\
7 & 1 & 0.543 & 0.030 \\
8 & 0.5 & 0.695 & 0.034 \\
\hline
\end{tabular}




\section{RESULTS AND DISCUSSION}

The normalized transverse response amplitude from Experiment $1(L / D=13)$ is presented in Figure 5. For purposes of validation, the normalized amplitude, $A_{y} / D$, from Experiment $1(L D=13)$ was compared with the results from Stappenbelt et al. [24], Morse et al. [25] and Khalak and Williamson [26]. Table 3 shows the parameters from the experiments used in this comparison. The selection of literature was based on the availability of a range of mass ratios and aspect ratios; however, the damping ratio in the present experiment is higher than those in the literature. As expected, the agreement with the literature was satisfactory for the particular $L / D$ investigated. It can be seen from Figure 5 that the motion amplitudes of the cylinder started at very low amplitude at the beginning of the experiment. As the velocity increased, the motion increased gradually to a peak value of 1.14 at a reduced velocity $U_{r}$ of 6.08 . The lock-in occurred and eased after the peak amplitude was reached before lock-out at around $U_{r}=10.42$. No hysteresis effect was observed since the data were collected by towing the cylinder from low to high velocities only. As mentioned before, Khalak and Williamson [26] captured a higher response and identified an upper branch for low mass ratio cylinders. However, in the present experiment at a low mass ratio $m^{*}$ of 1.6 only initial and lower branches were observed in the response plot. Although the present data showed a slightly higher peak amplitude than that in the literature, the initial and lower branch were well captured. It should be noted that the results on this plot published for comparative purposes were collected from a lower aspect ratio than in the present experiment (Table 3). Some of the data were collected from a different setup, e.g. a slender structure of infinite length with different end-conditions. This may have affected the results of the amplitude response. In addition to this, different parameters such as mass ratio and damping values used in these experiments may also have contributed to the variation of the plots. Nonetheless, the results obtained from Experiment $1(L / D=$ 13) show reasonably strong agreement with the literature.

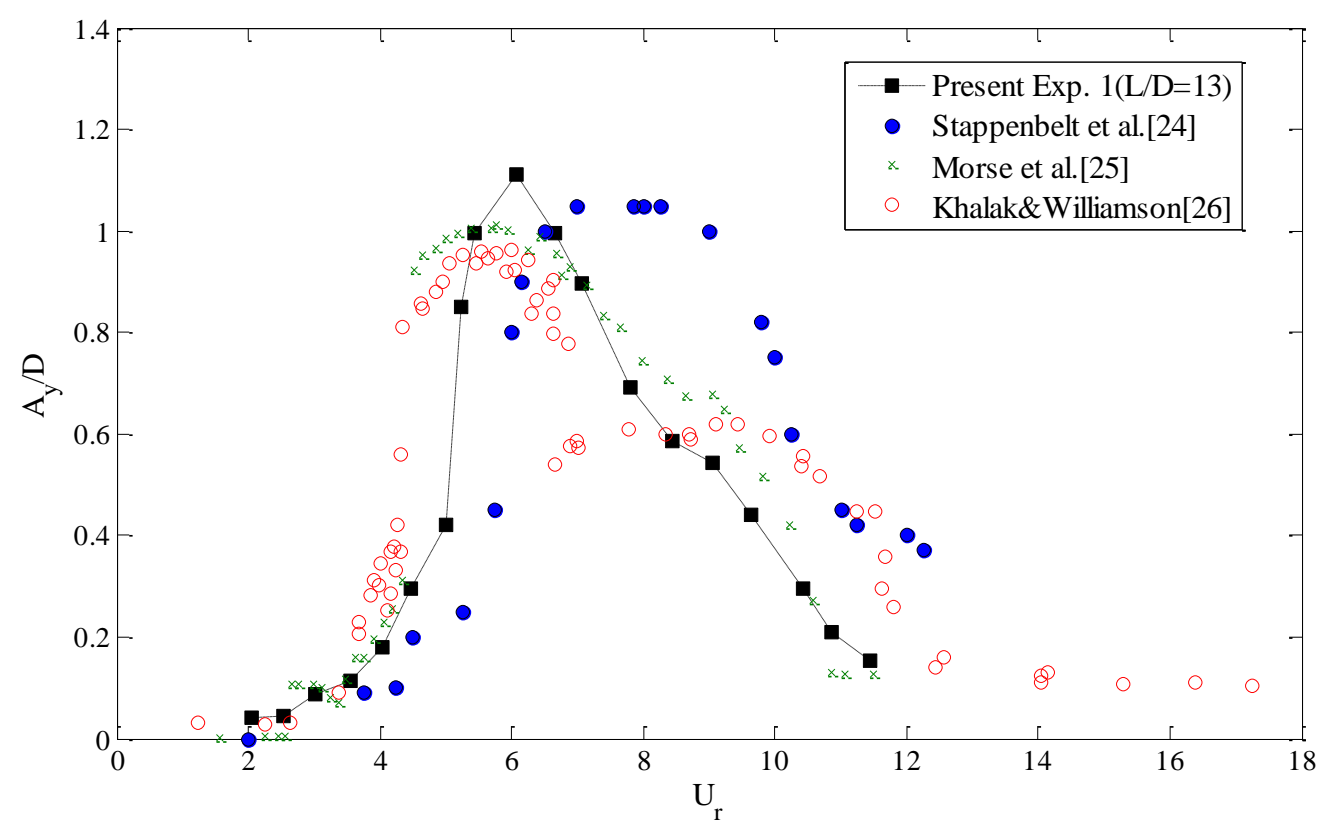

Figure 5. Normalized amplitude vs. reduced velocity for Experiment $1(L / D=13)$. 
Table 3. Comparison of the dimensionless parameters.

\begin{tabular}{llllc}
\hline Work & $m^{*}$ & \multicolumn{1}{c}{$\xi$} & $L / D$ & Endplate \\
\hline Present experiment & 1.6 & 0.031 & 13 & No \\
Stappenbelt et al. [24] & 2.36 & 0.006 & 12.27 & No \\
Morse et al. [25] & 9.3 & 0.0015 & 8 & No \\
Khalak\&Williamson [26] & 2.4 & 0.0059 & 8.5 & Yes \\
\hline
\end{tabular}

The vibration and vortex-shedding frequency plot from Experiment 1 are compared with Stappenbelt et al. [24] and Khalak and Williamson [26] in Figure 6. The agreement with the literature is good. The Strouhal numbers calculated from the gradient of vortex-shedding frequency curves are 0.15 (Experiment 1), 0.14 (Stappenbelt et al. [24]) and 0.18 (Khalak and Williamson [26]). The dependence of the Strouhal number on different aspect ratios will be discussed later in the context of the influence of aspect ratio on VIV response.

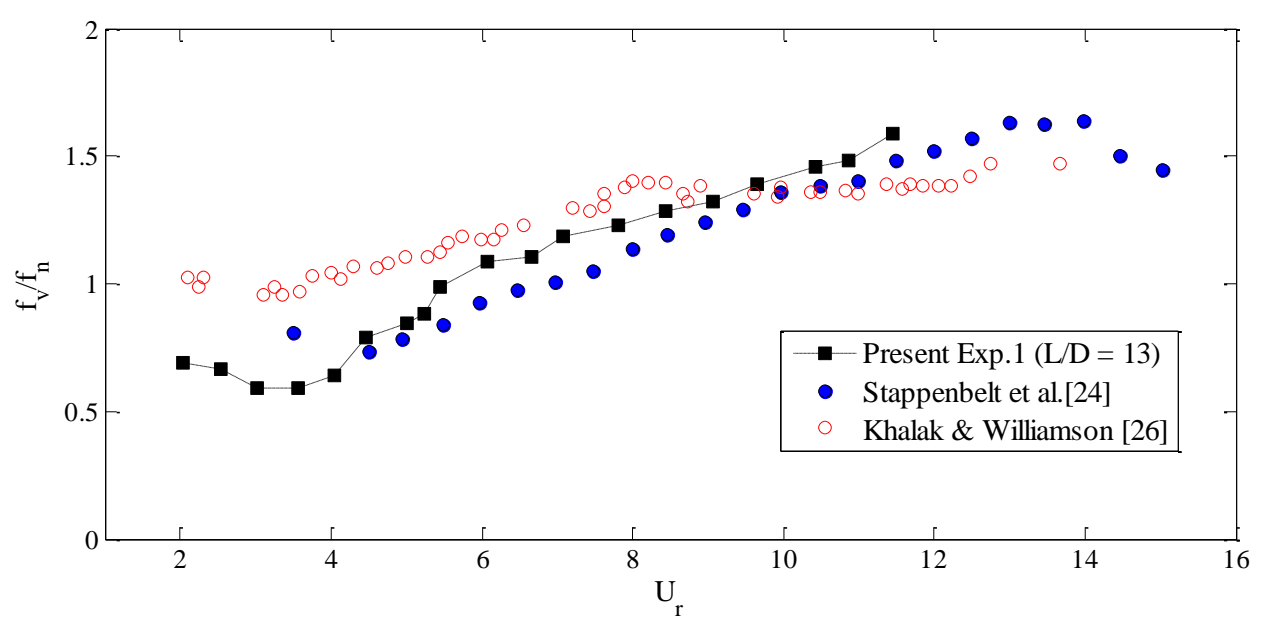

(a)



(b)

Figure 6. (a) Vibration frequency; (b) Vortex shedding frequency ratio for Experiment $1, m^{*}=1.6$, Stappenbelt et al. [24], $m^{*}=2.36$ and Khalak and Williamson [26], $m^{*}=$ 2.4 . 


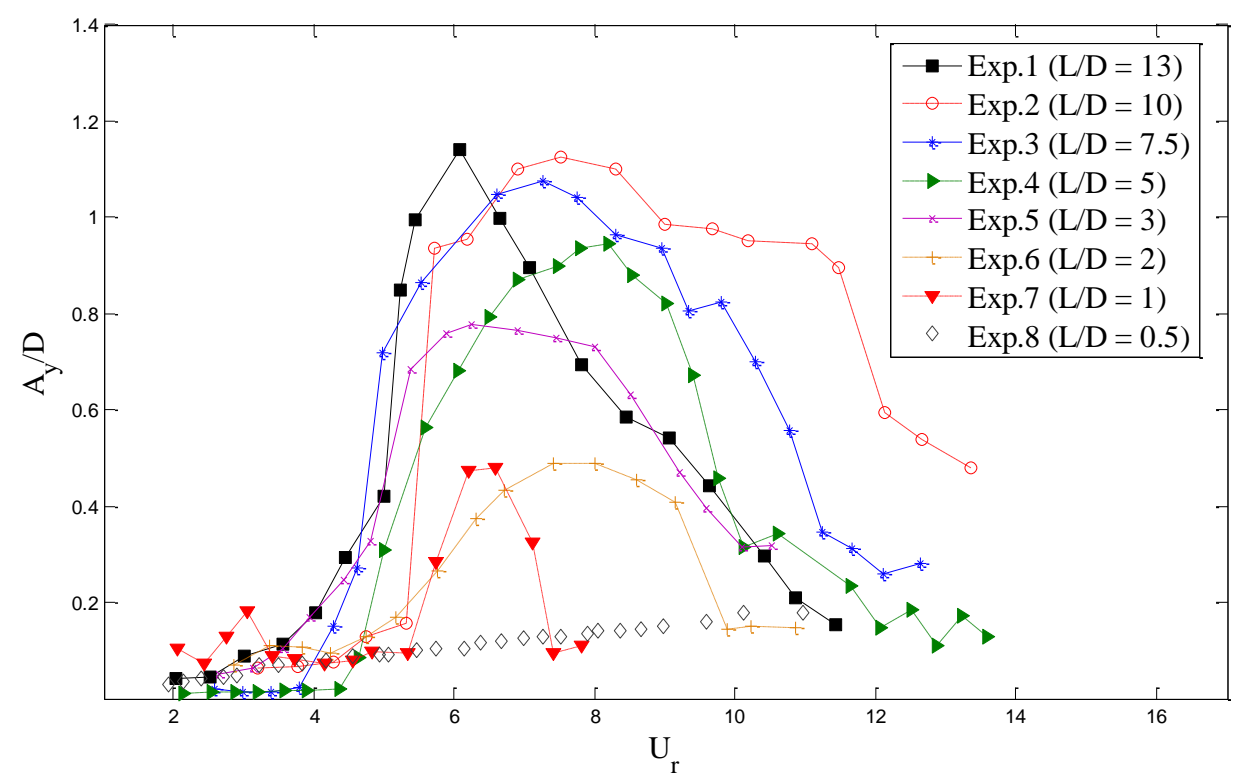

Figure 7. Normalized amplitude vs. reduced velocity for different aspect ratios.

Figure 7 shows the normalized amplitude response plots for each of the aspect ratios tested. As expected, the normalized amplitude response of the structure decreased with decreasing aspect ratio, which is consistent with Szepessy and Bearman [18] and Oouda [27]. The amplitude response for Experiment 1, which had the highest aspect ratio, showed the highest peak of all cases, and the response amplitude decreased as the aspect ratio decreased. The range of the VIV response was also observed generally to narrow down as the aspect ratio decreased. It is evident from Figure 7 that the aspect ratio significantly influenced the vibration response and synchronization range. The maximum amplitude responses were plotted against the aspect ratio to draw the relationship established as illustrated in Figure 8. Similarly to the previous plot, the value of the maximum response amplitude was observed to decrease as the aspect ratio decreased. There were slight differences between the high aspect ratio, $L / D=13$ to $L / D$ $=7.5$, and then a gradual decrease in aspect ratio lower than $L / D=7$. This is one of the key findings of this investigation. The vibration of the structures was expected to reduce as the aspect ratio reduced. The combined effect of the aspect ratio, the end conditions and the free surface affects the correlation length of the vortex formation, reducing the vibration of the structure [28]. In experiments simulating infinite length cylinders, the free surface and end conditions are eliminated by means of endplates or wall channels. Thus, the results would significantly differ from those obtained for a cylinder subjected to 3D end and surface effects [11].

The drag coefficients and lift coefficients obtained from the experiments are illustrated in Figure 9 and Figure 10 respectively. The hydrodynamic responses were observed to follow broadly similar trends to those of the amplitude response. The maximum value for each case was found to occur near the corresponding maximum amplitude response, as shown in Figure 7. Observations of the lock-in range comprised vortex shedding, vibration and natural frequencies during VIV. The lock-in was defined as the synchronization of the vortex shedding frequency and the vibration frequency over the reduced velocities. Figure 11 shows the variation of the frequency ratio $\left(f / f_{n}\right)$ over reduced velocity for the aspect ratio tested. The lock-in of VIV started at different reduced velocities for each case. The synchronization range varied depending on the 
aspect ratio. From the frequency response plot, it is apparent that the width of the lockin region decreases with reduced aspect ratio, for example from 3.56 to 10.42 at $L / D=$ 13 to 5.6 to 11.65 at $L / D=5$, and to 3.42 to 7.12 at $L / D=1$, as shown in Figure 11 . Note that the decrease of the lock-in width is also owed to the linear stiffness of the structure. The stiffness proportionally decreased over the aspect ratio and the lock-in range. The widest lock-in range was captured by Experiment 2 where the lock-out happened at $U_{r}=12.67$. The widening of the lock-in range leads to the shifting of the features of the amplitude response plot to higher reduced velocities. Experiment 7 showed the shortest lock-in range. This was owed to a lowered vortex shedding frequency that shortened the width of the lock-in region. The shortened width of the lock-in leads to a lower amplitude response of the structure as shown in the amplitude response plot.

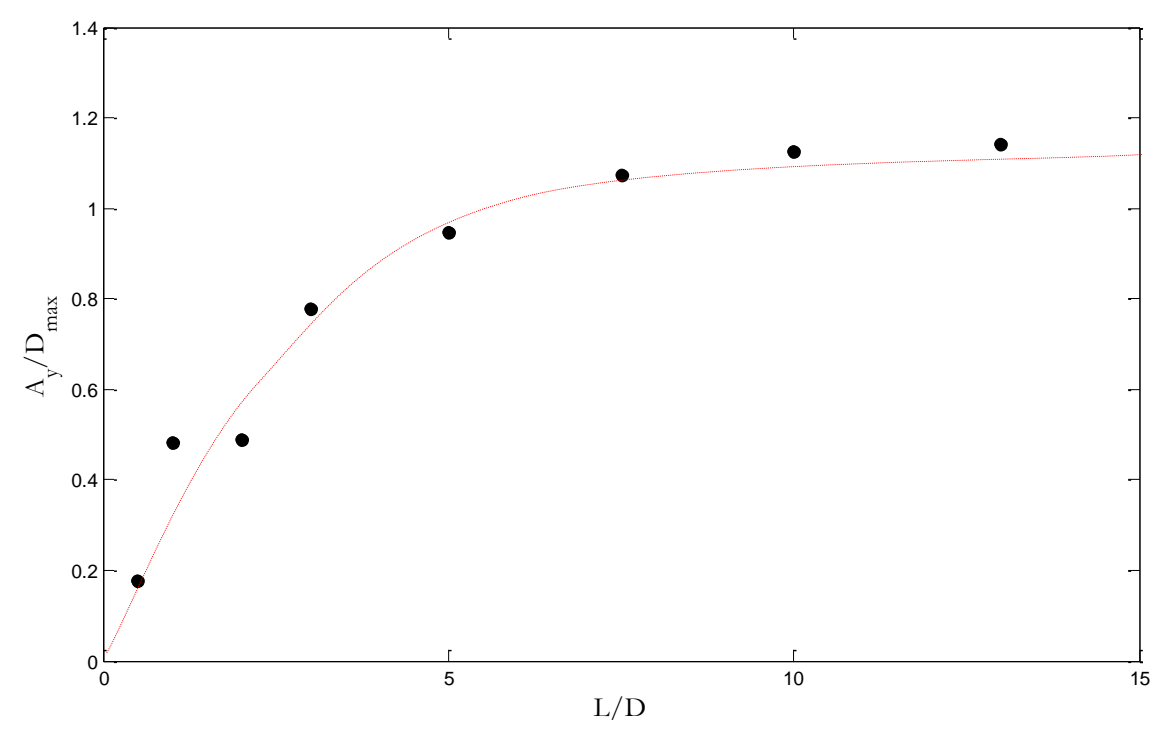

Figure 8. Maximum normalized amplitude vs. aspect ratio $(L / D)$.

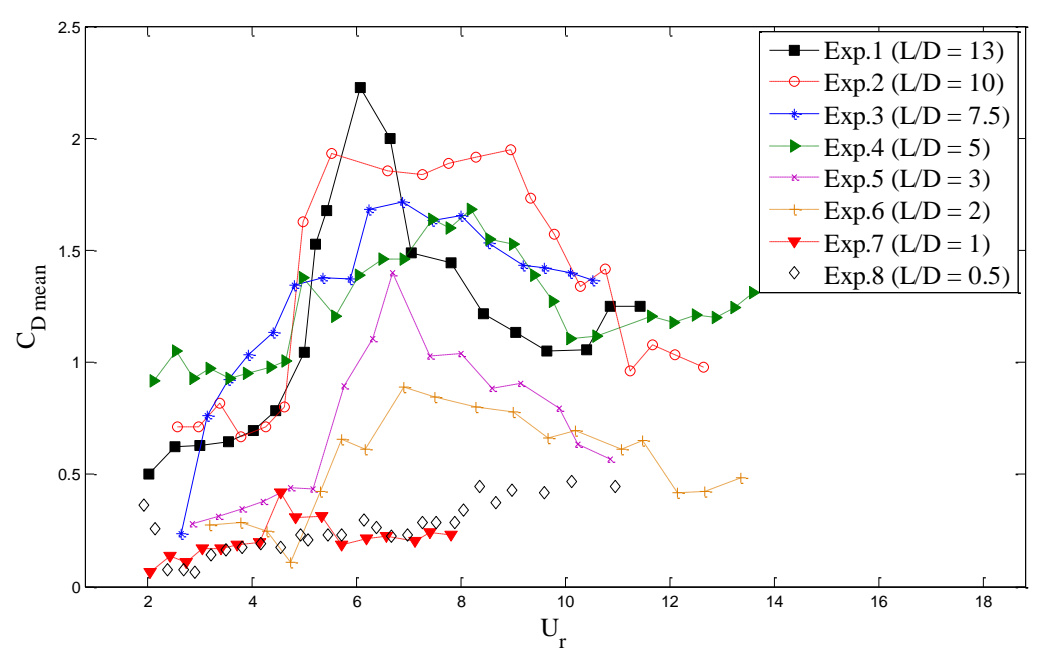

Figure 9. Mean drag coefficient vs. reduced velocity for different aspect ratios. 


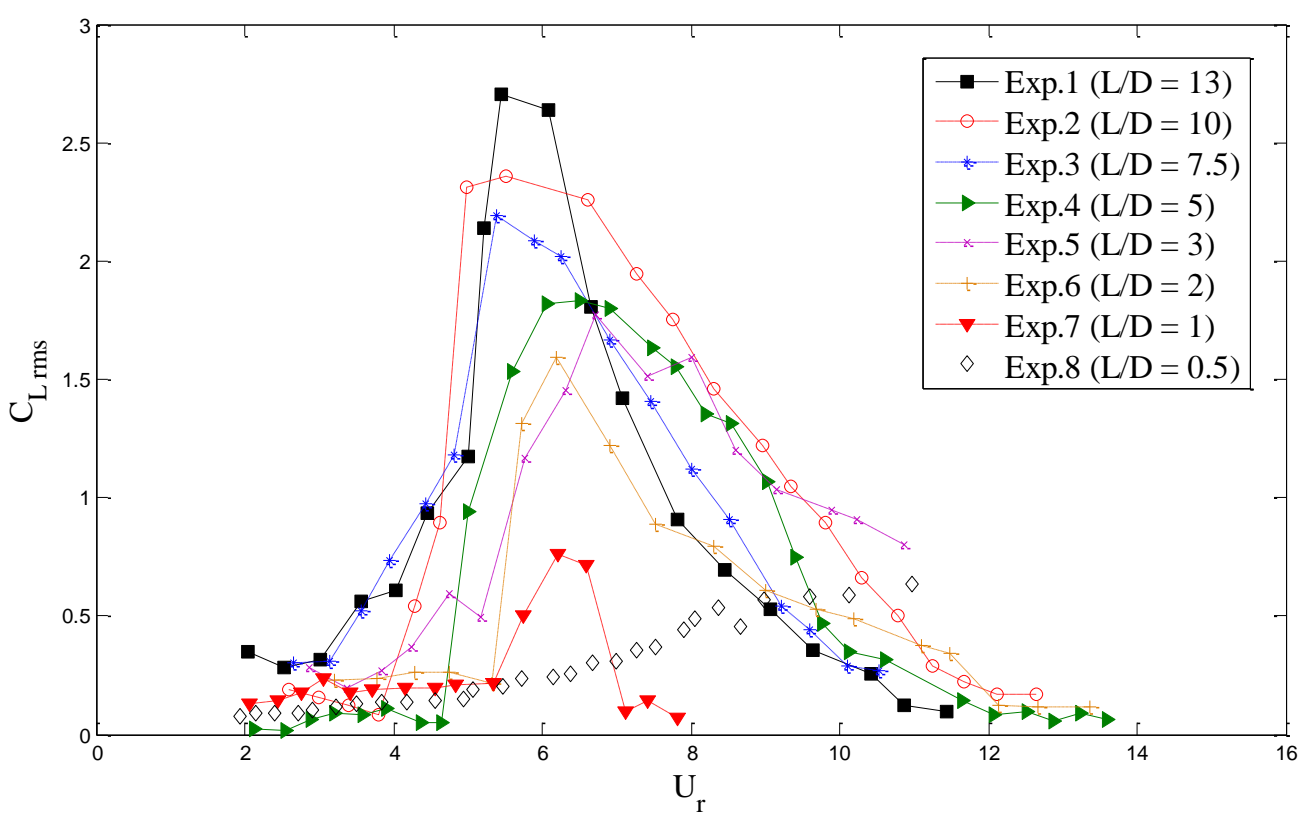

Figure 10. RMS lift coefficient vs. reduced velocity for different aspect ratios.

In a few cases, such as Experiments 2 and 4, lock-ins were observed to be delayed. The need for high energy achieved at higher flow velocities could be the reason for the delay in lock-in. The end conditions may contribute to the event as well. Additionally, it can be expected that the disturbance of the flow in the wake region reduced the correlation length, delaying the synchronization of the natural frequency and vibration frequencies. It can be seen from Figure 11 that synchronization was not observed in Experiment 8 ; in the aspect ratio of 0.5 the vibration frequency and vortex shedding frequency were not in agreement with each other throughout the range of reduced velocities. This is consistent with the earlier expectation on the responses, where the VIV was fully disturbed by the flow for a very low aspect ratio cylinder. Table 4 shows the summary of the excitation region for all aspect ratios examined. In a generic body, changes in aerodynamic behavior correspond to changes in the Strouhal number [29]. The Strouhal number for each case in this study was measured as the gradient of the vortex-shedding frequency curve. An example of a linear fit whose slope corresponded to the $S t$ was presented in Figure 11 for Experiment 1 from which $S t=$ 0.15 was obtained. This value was significantly lower than the values published by Roshko [30] for an infinite length cylinder with $S t=0.2$ to 0.21 . This lower value was perhaps because of a significantly low aspect ratio of 13 used, which allows for the result to be affected by the free surface and the end conditions. Work by Sheridan [31] and Rahman et al. [23] showed that a small change of distance between the cylinder and free surface could alter the near wake patterns that could also lead to a decrease in vibration frequency, $f_{v}$. Additionally, the vortex shedding frequency decreased throughout the synchronization and led to a reduction in the Strouhal number.

Some reduction St could be because the wake became three-dimensional in this work. It is clear from Figure 11 that the wake correlation length decreases when the aspect ratio decreases. The vortex formations along the cylinder span are functions of Reynolds number, amplitude, and turbulence intensity [11]. The amplitude of the vibration in the spanwise direction is supposed to increase the correlation length, as investigated by Blackburn and Melbourne [32]. However, as the flow velocity increases, 
the flow becomes turbulent, resulting in disturbance of the vortex formation and hence reducing the amplitude of vibration and the shedding frequencies.
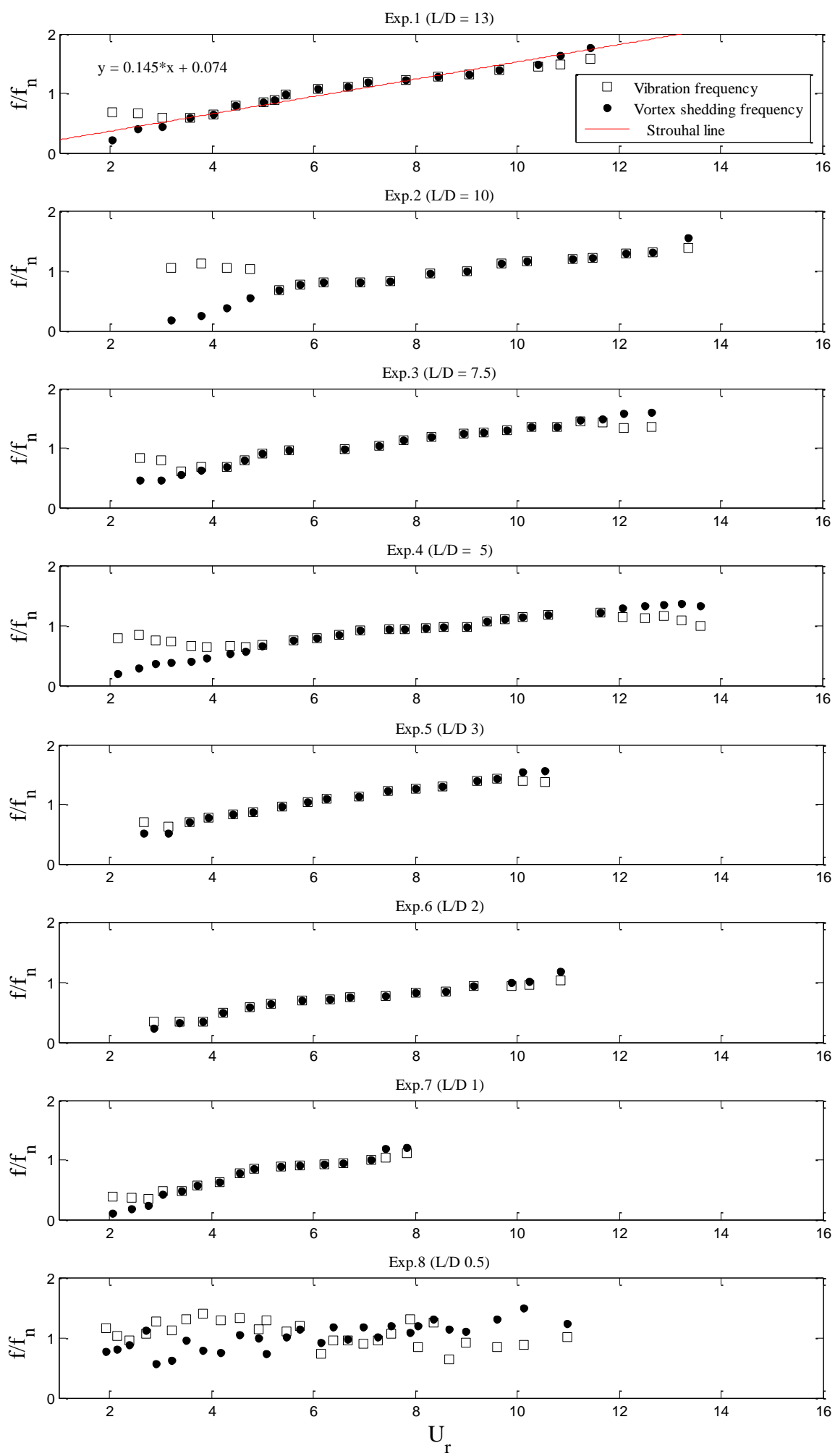

Figure 11. Frequency response vs. reduced velocity for different aspect ratios. 
Table 4. Synchronization points and the excitation range.

\begin{tabular}{|c|c|c|c|c|c|}
\hline $\begin{array}{l}\text { Exp. } \\
\text { no. }\end{array}$ & $\begin{array}{l}\text { Aspect ratio } \\
(L / D)\end{array}$ & $\begin{array}{l}\text { Lock-in point } \\
\left(U_{r}\right)\end{array}$ & $\begin{array}{l}\text { Lock-out } \\
\left(U_{r}\right)\end{array}$ & $\begin{array}{l}\text { Excitation } \\
\text { range }\end{array}$ & $S t$ \\
\hline 1 & 13 & 3.56 & 10.42 & 6.86 & 0.146 \\
\hline 2 & 10 & 5.32 & 12.67 & 7.35 & 0.130 \\
\hline 3 & 7.5 & 4.28 & 10.77 & 6.49 & 0.111 \\
\hline 4 & 5 & 5.60 & 11.65 & 6.05 & 0.098 \\
\hline 5 & 3 & 3.57 & 9.60 & 6.03 & 0.111 \\
\hline 6 & 2 & 3.83 & 9.15 & 5.32 & 0.100 \\
\hline 7 & 1 & 3.42 & 7.12 & 3.7 & 0.091 \\
\hline 8 & 0.5 & NA & NA & NA & 0.067 \\
\hline
\end{tabular}

The Strouhal numbers for each aspect ratio experiment are plotted in Figure 12. The gradient (Strouhal number) values from the plot increase as the aspect ratio increases. The value of the Strouhal number has been compared with the work done by Oouda [27]. The trends of the present experiments and Oouda [27] appear to be continuous, thus indicating a common underlying flow physics. There is a monotonic increase in St with $L / D$, which asymptotes to a constant value of 0.2 as seen in Oouda's results. The lowest St of 0.067 was recorded in the present experiment at $L / D$ of 0.5 (Table 4). The highest $L / D$ value of the present work coincides with the lowest aspect ratio used by Oouda [27] and thus gives a more complete picture of the effect of $L / D$ on St. A reduction of more than $10 \%$ in St value corresponding to those at high aspect ratios indicates that ignoring $L / D$ effects on St may lead to a wrong prediction of the vortex shedding frequency for short cylinders and hence lead to unconservative fatigue life estimation of mooring line components and offshore structures.

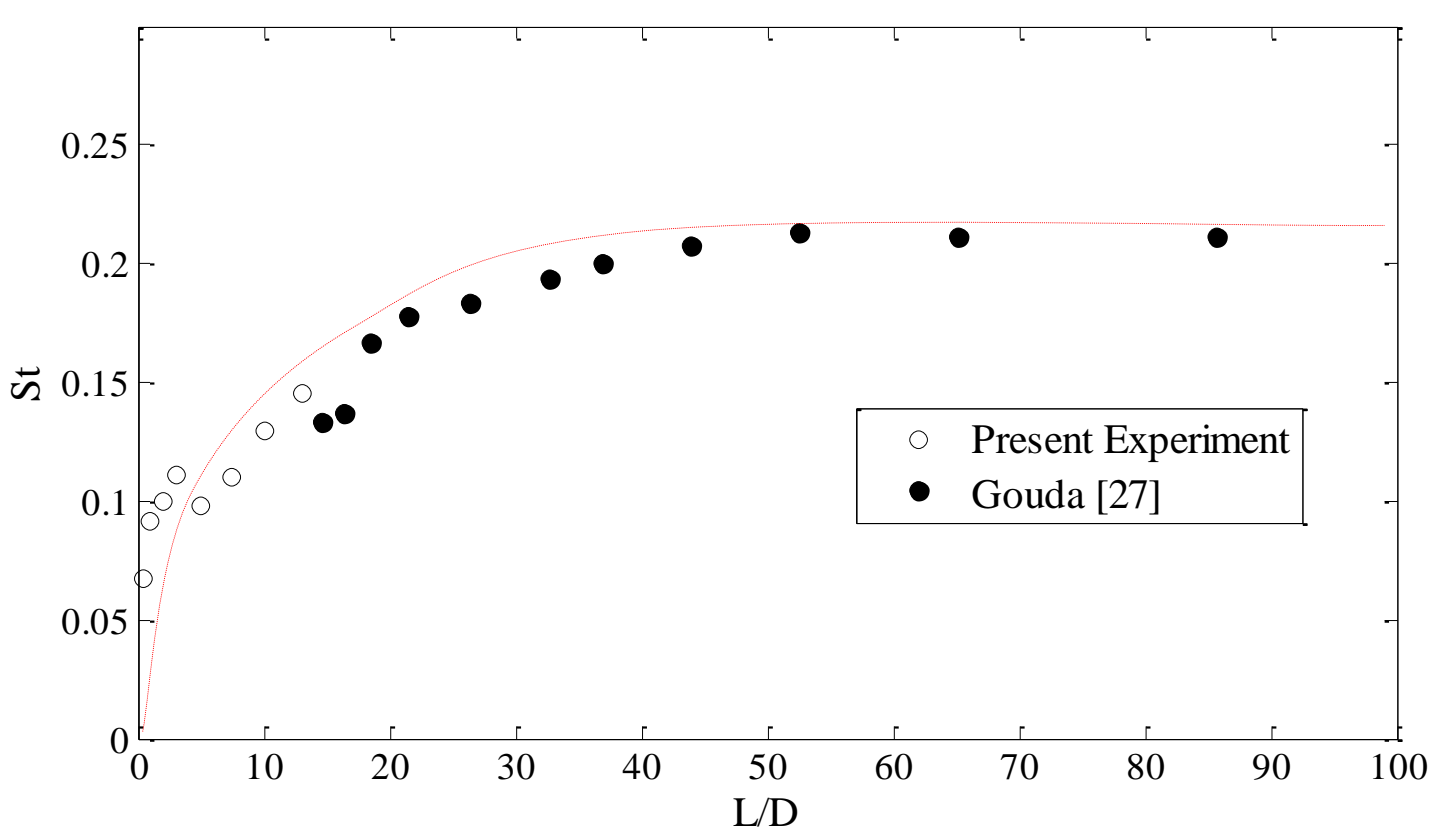

Figure 12. Strouhal number vs. aspect ratio. 
Notably, in Figure 12, the decrease was pronounced as the aspect ratio decreased from $L / D=50$. The fact that Strouhal numbers continue to decrease as the aspect ratio decreases is consistent with the earlier hypothesis on the effect of aspect ratio on the VIV phenomenon. It should be noted that the decrease in the vortex-shedding frequency may be related to changes in the correlation length of vortex formations. The vortex formations along the cylinder span are a function of Reynolds number, amplitude and turbulence intensity, as discussed by [11]. The amplitude of the vibration in the spanwise direction is assumed to increase the correlation length [32]. However, as the flow increases in velocity, it becomes turbulent, disturbing vortex formation and reducing the amplitude of vibration and shedding frequencies.

\section{CONCLUSIONS}

In this paper, eight cases of variable aspect ratio have been studied. Data collected from the experiments show substantial agreement with the literature. It can be inferred from the experimental works that as the aspect ratio reduces VIV reduces and completely diminishes as the aspect ratio reduces to $L / D=0.5$. This abrupt variation was similar to the mean drag and RMS lift coefficients, which followed the same trend with the decrease of the cylinder aspect ratio. In terms of frequencies, it appears that the Strouhal numbers have lower values of $S t<0.15$ compared with the published values of 0.2 to 0.21 for stationary smooth cylinders with a subcritical Reynolds number. These lower values of St corresponded to the behavior of the lock-in for each aspect ratio examined. The three-dimensional effects of the end condition strongly influenced the wake correlation length, which decreased since the vortex shedding was disturbed by the asymmetric flow.

\section{ACKNOWLEDGEMENTS}

The authors would like to express their gratitude to University of Western Australia for providing laboratory facilities and support.

\section{REFERENCES}

[1] King R. A review of vortex shedding research and its application. Ocean Engineering. 1977;4:141-71.

[2] Sarpkaya T. Fluid forces on oscillating cylinders. NASA STI/Recon Technical Report A. 1978;78:46523.

[3] Sarpkaya T. A critical review of the intrinsic nature of vortex-induced vibrations. Journal of Fluids and Structures. 2004;19:389-447.

[4] Griffin OM. Otec cold water pipe design for problems caused by vortex-excited oscillations. Ocean Engineering. 1981;8:129-209.

[5] Bearman PW. Vortex shedding from oscillating bluff bodies. Annual Review of Fluid Mechanics. 1984;16:195-222.

[6] Pantazopoulos MS. Vortex-induced vibration parameters: critical review. USA: American Society of Mechanical Engineers; 1994.

[7] Williamson C, Govardhan R. Vortex-induced vibrations. Annual Review of Fluid Mechanics. 2004;36:413-55. 
[8] Williamson CHK, Govardhan R. A brief review of recent results in vortexinduced vibrations. Journal of Wind Engineering and Industrial Aerodynamics. 2008;96:713-35.

[9] Chen SS. Flow-induced vibration of circular cylindrical structures. Master Thesis, The University of Chicago, USA; 1987.

[10] Blevins RD. Flow-induced vibration. Classifications and Lessons from Practical Experiences. Edited by Kaneko S, Nakamura T, Inada F, Kato M, Mureithi NW. 1990.

[11] Sumer BM, Fredsøe J. Hydrodynamics around cylindrical structures: World Scientific; 1997.

[12] Chakrabarti S. Handbook of Offshore Engineering (2-volume set): Elsevier; 2005.

[13] Major F, Eggan S. Significant benefits with a cylindrical hull for FPSO, drilling, LNG, and offshore power plant applications. Petrotech, Delhi. 2009.

[14] Nishioka M, Sato H. Measurements of velocity distributions in the wake of a circular cylinder at low Reynolds numbers. Journal of Fluid Mechanics. 1974;65:97-112.

[15] West G, Apelt C. The effects of tunnel blockage and aspect ratio on the mean flow past a circular cylinder with Reynolds numbers between 104 and 105 . Journal of Fluid Mechanics. 1982;114:361-77.

[16] Lee T, Budwig R. A study of the effect of aspect ratio on vortex shedding behind circular cylinders. Physics of Fluids A: Fluid Dynamics (1989-1993). 1991;3:309-15.

[17] Baban Ft, So R. Aspect ratio effect on flow-induced forces on circular cylinders in a cross-flow. Experiments in Fluids. 1991;10:313-21.

[18] Szepessy S, Bearman P. Aspect ratio and end plate effects on vortex shedding from a circular cylinder. Journal of Fluid Mechanics. 1992;234:191-217.

[19] Albarède P, Monkewitz PA. A model for the formation of oblique shedding and "chevron' patterns in cylinder wakes. Physics of Fluids A: Fluid Dynamics (1989-1993). 1992;4:744-56.

[20] Norberg C. An experimental investigation of the flow around a circular cylinder: influence of aspect ratio. Journal of Fluid Mechanics. 1994;258:287316.

[21] Nakamura A, Okajima A, Kosugi T. Experiments on flow-induced in-line oscillation of a circular cylinder in a water tunnel (2nd report, influence of the aspect ratio of a cantilevered circular cylinder): Flow-induced vibration of bluff bodies. JSME International Journal Series B, Fluids and Thermal Engineering. 2001;44:705-11.

[22] Gonçalves RT, Fujarra AL. Experimental study on vortex-induced vibration of floating circular cylinders with low aspect ratio. ASME 2014 33rd International Conference on Ocean, Offshore and Arctic Engineering: American Society of Mechanical Engineers; 2014; V002T08A21-VT08A21.

[23] Rahman MA, Esakki M, Kamarudin MH, Thiagarajan KP. Free surface effects on vortex induced vibrations of cylindrical offshore structures. Proceedings of the 6th Australasian Congress on Applied Mechanics. 2010; 936-43.

[24] Stappenbelt B, Lalji F, Tan G. Low mass ratio vortex-induced motion. 16th Australasian Fluid Mechanics Conference. 2007; 1491-7. 
[25] Morse TL, Govardhan RN, Williamson CHK. The effect of end conditions on the vortex-induced vibration of cylinders. Journal of Fluids and Structures. 2008;24:1227-39.

[26] Khalak A, Williamson CHK. Motions, forces and mode transitions in vortexinduced vibrations at low mass-damping. Journal of Fluids and Structures. 1999;13:813-51.

[27] Oouda B. Some measurements of the phenomena of vortex shedding and induced vibrations of circular cylinder. Technische Universitat Berlin Report DLR-FB. 1975:75-01.

[28] Rahman MAA, Leggoe J, Thiagarajan K, Mohd MH, Paik JK. Numerical simulations of vortex-induced vibrations on vertical cylindrical structure with different aspect ratios. Ships and Offshore Structures. 2015:1-19.

[29] Pastò S. Vortex-induced vibrations of a circular cylinder in laminar and turbulent flows. Journal of Fluids and Structures. 2008;24:977-93.

[30] Roshko A. Experiments on the flow past a circular cylinder at very high Reynolds number. Journal of Fluid Mechanics. 1961;10:345-56.

[31] Sheridan J, Lin JC, Rockwell D. Flow past a cylinder close to a free surface. Journal of Fluid Mechanics. 1997;330:1-30.

[32] Blackburn HM, Melbourne WH. Sectional lift forces for an oscillating circular cylinder in smooth and turbulent flows. Journal of Fluids and Structures. 1997;11:413-31. 